\title{
Altered expression of pannexin proteins in patients with temporal lobe epilepsy
}

\author{
TING JIANG, HONGYU LONG, YAN MA, LILI LONG, YI LI, \\ FENG LI, PINTING ZHOU, CHUNYUN YUAN and BO XIAO
}

\begin{abstract}
Department of Neurology, Xiangya Hospital, Central South University, Changsha, Hunan 410008, P.R. China
\end{abstract}
Received May 28, 2013; Accepted October 7, 2013

DOI: $10.3892 / \mathrm{mmr} .2013 .1739$

\begin{abstract}
The aim of the present study was to investigate the expression of the pannexin (Panx) proteins, Panx1 and Panx2, in the temporal lobe tissue of patients with temporal lobe epilepsy (TLE). Immunohistochemistry and western blotting methods were used to localize and quantify Panx1 and Panx2 in the surgically removed brain tissue of patients with TLE $(n=37)$. The results were then compared with non-epileptogenic controls $(n=9)$. Panx 1 and Panx 2 expression was detected in the temporal lobe cortex of patients with TLE and in the control tissues. Panx1 and Panx 2 proteins were expressed in all layers of the epileptic cortex, but predominantly in layers II and III of the cortex in the control group. Panx1 protein expression was significantly higher in the temporal lobe cortex of the patients with TLE than in the controls $(\mathrm{P}<0.05$; t-test); however, no significant differences were identified in the Panx2 expression levels between the patients and the controls ( $P>0.05$; t-test). The expression of the two Panx proteins in the tissue layers of the epileptic cortex varied in the patients and controls. The results indicate that Panx channels may be involved in the pathogenesis of TLE.
\end{abstract}

\section{Introduction}

Temporal lobe epilepsies (TLEs) are a group of medical disorders that result in recurrent epileptic seizures arising from one or both temporal lobes of the brain.

The presence of gap junction proteins termed pannexins (Panx) was first reported by Panchin et al in 2000 (1). These proteins release large signaling molecules, including ATP and arachidonic acid derivatives. Three subtypes of the Panx family have been identified as Panx1, 2 and 3 (2,3). Human and mouse Panx1 mRNA is ubiquitously expressed in normal tissues of humans and mice, respectively; however, human Panx2 is a

Correspondence to: Dr Bo Xiao, Department of Neurology, Xiangya Hospital, Central South University, No. 87 Xiangya Road, Kaifu, Changsha, Hunan 410008, P.R. China

E-mail: xiaobo62_xy@126.com

Key words: pannexin, temporal lobe epilepsy brain-specific gene and Panx3 is expressed predominantly in osteoblasts and synovial fibroblasts in in silico evaluation. Furthermore, Panx1 has been shown to form functional hemichannels by itself or in conjunction with Panx2 $(1,4)$.

Panx1 channel opening has been indicated to contribute to epileptiform seizure activity. Thompson et al (5) identified that Panx1 hemichannel opening is triggered by $\mathrm{N}$-Methyl-D-aspartate stimulation and may be a significant target for the treatment of epilepsy. Furthermore, it has been demonstrated that reduced levels of extracellular glucose due to fasting or adhering to a ketogenic diet may induce Panx1 hemichannel-mediated ATP release from CA3 neurons (6). This in turn hyperpolarizes neuronal membrane potentials via ATP-sensitive potassium channels. The role of Panx 2 in the pathology of epilepsy is less certain than that of Panx1. However, since Panx 2 may participate in the functional hemichannels with Panx1, this subtype was also included in the present study in order to investigate whether the expression pattern changed in epileptic brain tissues.

To the best of our knowledge, the current study compared for the first time the expression pattern of Panx 1 and Panx 2 in brain tissues in patients with TLE and non-epileptic patients using immunohistochemistry and western blotting techniques, to aid in our further understanding of the pathogenesis of TLE.

\section{Patients and methods}

Patient selection. Epileptic brain tissue was obtained from 37 patients with TLE and from nine control patients with traumatic brain injury (Tables I and II) (7).

The patients with TLE had typical clinical manifestations and characteristic electroencephalography (EEG) findings. The diagnosis of the seizure type was confirmed according to the 1981 International Classification of Epileptic Seizures of the International League Against Epilepsy (8). Prior to surgery, the epileptic lesions were located in all patients by brain magnetic resonance imaging (MRI) and 24-h or video-EEG recordings. Sphenoidal electrode monitoring and intraoperative electrocorticography were performed to localize the epileptic lesion prior to resection.

For the control experiments, temporal lobe tissue from nine patients with traumatic brain injury was obtained from the files of the Department of Neurosurgery of Xiangya Hospital (Changsha, China). These patients had undergone surgery due 
Table I. Data for patients with temporal lobe epilepsy.

\begin{tabular}{|c|c|c|c|c|c|}
\hline Sample & Age (years) & Gender & Disease duration (years) & Lobectomy $^{\mathrm{a}}$ & Types of seizures \\
\hline E1 & 22 & M & 14 & $\mathrm{~L}$ & CPS, SGS \\
\hline E2 & 27 & M & 21 & $\mathrm{R}$ & CPS, SGS \\
\hline E3 & 28 & M & 12 & $\mathrm{R}$ & CPS, SGS \\
\hline E4 & 20 & M & 18 & $\mathrm{R}$ & SPS, SGS \\
\hline E5 & 27 & M & 4 & $\mathrm{R}$ & CPS \\
\hline E6 & 39 & $\mathrm{~F}$ & 37 & $\mathrm{R}$ & CPS, SGS \\
\hline E7 & 20 & $\mathrm{M}$ & 8 & $\mathrm{R}$ & CPS, SGS \\
\hline E8 & 30 & M & 18 & $\mathrm{~L}$ & CPS, SGS \\
\hline E9 & 28 & $\mathrm{~F}$ & 12 & $\mathrm{~L}$ & CPS, SGS \\
\hline E10 & 27 & M & 11 & $\mathrm{R}$ & SPS, SGS \\
\hline E11 & 32 & $\mathrm{~F}$ & 4 & $\mathrm{~L}$ & CPS \\
\hline E12 & 26 & M & 3 & $\mathrm{R}$ & CPS \\
\hline E13 & 17 & M & 7 & $\mathrm{~L}$ & SPS, SGS \\
\hline E14 & 22 & M & 18 & $\mathrm{R}$ & CPS \\
\hline E15 & 25 & M & 8 & $\mathrm{R}$ & GTCS, SPS \\
\hline E16 & 29 & M & 8 & $\mathrm{R}$ & GTCS, TS \\
\hline E17 & 32 & M & 4 & $\mathrm{~L}$ & CPS \\
\hline E18 & 20 & M & 8 & $\mathrm{R}$ & CPS, SGS \\
\hline E19 & 25 & M & 6 & $\mathrm{R}$ & GTCS, TS \\
\hline E20 & 17 & M & 4 & $\mathrm{~L}$ & CPS, SGS \\
\hline E21 & 24 & M & 3 & $\mathrm{~L}$ & SPS, SGS \\
\hline E22 & 23 & M & 20 & $\mathrm{~L}$ & CPS, SGS \\
\hline E23 & 29 & M & 25 & $\mathrm{R}$ & GTCS, SPS \\
\hline E24 & 30 & M & 2 & $\mathrm{~L}$ & CPS \\
\hline E25 & 18 & $\mathrm{~F}$ & 7 & $\mathrm{~L}$ & CPS \\
\hline E26 & 30 & $\mathrm{~F}$ & 12 & $\mathrm{R}$ & SPS, SGS \\
\hline E27 & 22 & M & 10 & $\mathrm{~L}$ & SPS, SGS \\
\hline E28 & 30 & M & 5 & $\mathrm{~L}$ & SPS, SGS \\
\hline E29 & 24 & $\mathrm{~F}$ & 14 & $\mathrm{R}$ & CPS, SGS \\
\hline E30 & 22 & M & 17 & $\mathrm{R}$ & SPS, SGS \\
\hline E31 & 32 & $\mathrm{~F}$ & 28 & $\mathrm{R}$ & SPS, SGS \\
\hline E32 & 23 & M & 12 & $\mathrm{R}$ & SPS, SGS \\
\hline E33 & 21 & M & 17 & $\mathrm{R}$ & CPS, SGS \\
\hline E34 & 35 & $\mathrm{~F}$ & 20 & $\mathrm{R}$ & CPS, SGS \\
\hline E35 & 31 & $\mathrm{~F}$ & 10 & $\mathrm{~L}$ & SPS, SGS \\
\hline E36 & 32 & M & 20 & $\mathrm{~L}$ & CPS, SGS \\
\hline E37 & 34 & $\mathrm{~F}$ & 21 & $\mathrm{~L}$ & SPS, SGS \\
\hline
\end{tabular}

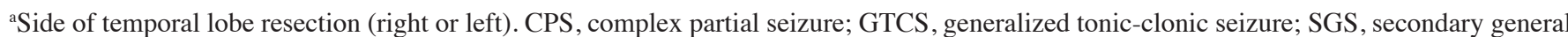
seizure; T, tonic seizures; SPS, simple partial seizure.

to severe brain trauma and had no history of epilepsy or other abnormal pathology and exposure to anti-epileptic drugs. The samples comprised temporal neocortical tissue adjacent to the trauma-induced lesion.

Written informed consent was obtained from the patients or their relatives with regard to the use of data and tissues for the purpose of research studies. The study complied with guidelines for the conduct of research involving human subjects as established by the National Institute of Health and the Committee on Human Research at Xiangya Hospital.
Tissue processing. The brain tissue from the epileptic patients and the patients with traumatic brain injury was obtained during surgery. Following dissection, the specimens were divided into two portions. One portion was immediately frozen in liquid nitrogen, stored at $-80^{\circ} \mathrm{C}$ and subsequently used for western blotting. The other portion was fixed in $4 \%$ paraformaldehyde, embedded in paraffin blocks, cut into 5.0- $\mu \mathrm{M}$ transverse sections on a Model RM2135 microtome (Leica, Wetzlar, Germany) and preserved for future use at room temperature. 
Table II. Data for patients used as controls.

\begin{tabular}{lccccc}
\hline Sample & Age (years) & Gender & Lobectomy $^{\text {a }}$ & Etiological diagnosis & Adjacent tissue pathology \\
\hline C1 & 39 & M & L & Trauma & Normal \\
C2 & 32 & F & R & Trauma & Normal \\
C3 & 28 & M & R & Trauma & Normal \\
C4 & 40 & M & L & Trauma & Normal \\
C5 & 22 & F & R & Trauma & Normal \\
C6 & 27 & M & L & Trauma & Normal \\
C7 & 21 & M & R & Trauma & Normal \\
C8 & 10 & M & L & Trauma & Normal \\
C9 & 34 & F & R & &
\end{tabular}

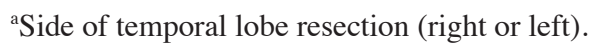

Immunohistochemistry. Temporal lobe tissue sections of patients with epilepsy were processed for Panx immunohistochemistry using avidin-biotin peroxidase methods. Following dewaxing and rehydration, endogenous peroxidases were inactivated by adding $1 \% \mathrm{H}_{2} \mathrm{O}_{2}$ for $30 \mathrm{~min}$. The sections were rinsed in $0.01 \mathrm{M}$ phosphate-buffered saline (PBS) and incubated in $10 \%$ normal goat serum in PBS for $2 \mathrm{~h}$ to reduce any non-specific binding.

The sections were then incubated overnight with a Panx antiserum at $4^{\circ} \mathrm{C}$. Panx1 (1:150, rabbit polyclonal antibody; Abcam, Cambridge, UK) and Panx2 (1:250, rabbit polyclonal antibody; Abcam) antisera were diluted in PBS containing 2\% normal goat serum. Following rinsing, the sections were incubated in biotinylated secondary antiserum (rabbit anti-goat IgG, 1:200; Vector Laboratories, Burlingame, CA, USA) at room temperature for $1 \mathrm{~h}$. Following a further rinsing, the sections were incubated in avidin-biotin peroxidase complex (1:200; Vectastain Elite ABC; Vector Laboratories) in PBS for $1 \mathrm{~h}$.

To visualize the peroxidase labeling, the sections were processed with $0.06 \%$ diaminobenzidine tetrahydrochloride and $0.006 \% \mathrm{H}_{2} \mathrm{O}_{2}$ diluted in $0.075 \mathrm{M}$ PBS for $10 \mathrm{~min}$. Following rinsing, the sections were dehydrated and placed under a cover slip. Visual field images were obtained layer by layer from every section using an Nikon TE2000 automatic microscope (Nikon, Tokyo, Japan) and Nikon DS-Fi1 (Nikon) pathology system. The full featured photo of the cortex was captured by PT Gui Pro 9.0.4 (New House Internet Services B.V., Rotterdam, The Netherlands).

Western blotting. A western blot analysis was performed to compare Panx immunoreactivity in the TLE and control groups. Tissue samples were cut into small sections, homogenized in radio-immunoprecipitation assay lysis buffer $[0.05 \mathrm{~mol} / 1$ Tris-HCl (pH 7.4), $0.15 \mathrm{~mol} / 1 \mathrm{NaCl}, 1 \%$ Triton X-100, $1 \%$ (w/v) sodium deoxycholate and $0.1 \%$ sodium dodecyl sulfate (SDS)], including $1 \mathrm{mM}$ phenylmethylsulfonyl fluoride, and centrifuged at $12,000 \mathrm{x} \mathrm{g}$ at $4^{\circ} \mathrm{C}$ for $5 \mathrm{~min}$. The protein concentration of the lysates was determined using a bicinchoninic acid protein assay kit (Pierce Biotechnology, Rockford, IL, USA). The extracts $(40 \mu \mathrm{g})$ were resolved by $10 \%$ SDS-polyacrylamide gel electrophoresis and electrotransferred to a polyvinylidene difluoride (PVDF) membrane. The
PVDF membranes were divided into two sections according to the location of molecular weight markers to detect Panx2 (69 kDa) and $\beta$-actin (42 kDa), which was used as a loading control. The PVDF membrane was blocked with $5 \%$ milk prepared in tris-buffered saline with $0.05 \%$ Tween-20 (TBST) then incubated for $1 \mathrm{~h}$ at room temperature.

Following extensive washing with TBST, the membranes were incubated in Panx2 (1:200, rabbit polyclonal antibody, Abcam, UK) and $\beta$-actin (mouse monoclonal IgG, 1:1,000; Santa Cruz Biotechnology, Santa Cruz, CA, USA) antibodies for $12 \mathrm{~h}$ at $4^{\circ} \mathrm{C}$. Following three 10 -min washes with TBST, each blot was incubated with a secondary antibody (goat anti-rabbit IgG for Panx2 blot and goat anti-mouse IgG for $\beta$-actin blot; Beyotime Company, Haimen, China) at a dilution of 1:1,000 for $1 \mathrm{~h}$. Following three further washes with TBST and one wash with TBS (15 min each), the Panx 2 protein and $\beta$-actin were detected using an enhanced chemiluminescence detection kit according to the manufacturer's instructions (Thermo Scientific Pierce, Waltham, MA, USA).

Panx1 was assayed by incubating the PVDF membranes with $\beta$-actin in stripping buffer [700 $\mu 114.4 \mathrm{mmol} / 1$ $\beta$-mercaptoethanol, SDS $1 \mathrm{~g}, 0.5 \mathrm{~mol} / 1$ Tris-HCl (pH 6.8) and $12.5 \mathrm{ml}$ in $100 \mathrm{ml}$ ultra-pure water] for $30 \mathrm{~min}$. The blot was then re-hybridized with the anti-Panx1 primary antibody (1:100, rabbit polyclonal antibody; Abcam) for $12 \mathrm{~h}$ at $4^{\circ} \mathrm{C}$. Following washing with TBST three times, the blot was incubated with goat anti-rabbit IgG (1:1000, Beyotime Company). Following washing three times with TBST and once with TBS, the Panx1 level was detected by enhanced chemiluminescence as described.

Statistical analysis. Statistical analysis was performed using SPSS (version 17.0; SPSS, Inc., Chicago, IL, USA). Data were presented as the mean \pm SD and any differences between active and control groups were determined using $\chi^{2}$ tests and t-tests. $\mathrm{P}<0.05$ was considered to indicate a statistically significant difference.

\section{Results}

Patient characteristics. No significant differences were identified in the ratios of age, gender and lobectomy among 
Table III. Clinical characteristics of the subjects.

\begin{tabular}{lccc}
\hline & TLE group & Control group & P-value \\
\hline Age & $26.29 \pm 5.33$ & $28.11 \pm 9.53$ & $0.443^{\mathrm{b}}$ \\
Gender & & & \\
Male & 27 & 6 & $0.706^{\mathrm{c}}$ \\
Female & 10 & 3 & \\
Lobectomy & & & \\
Left & 16 & 4 & $0.948^{\mathrm{c}}$ \\
Right & 21 & 5 & \\
\hline
\end{tabular}

${ }^{\text {aSide }}$ of temporal lobe resection (right or left); ${ }^{b}$-test; ${ }^{c} \chi^{2}$ test. TLE, temporal lobe epilepsy.

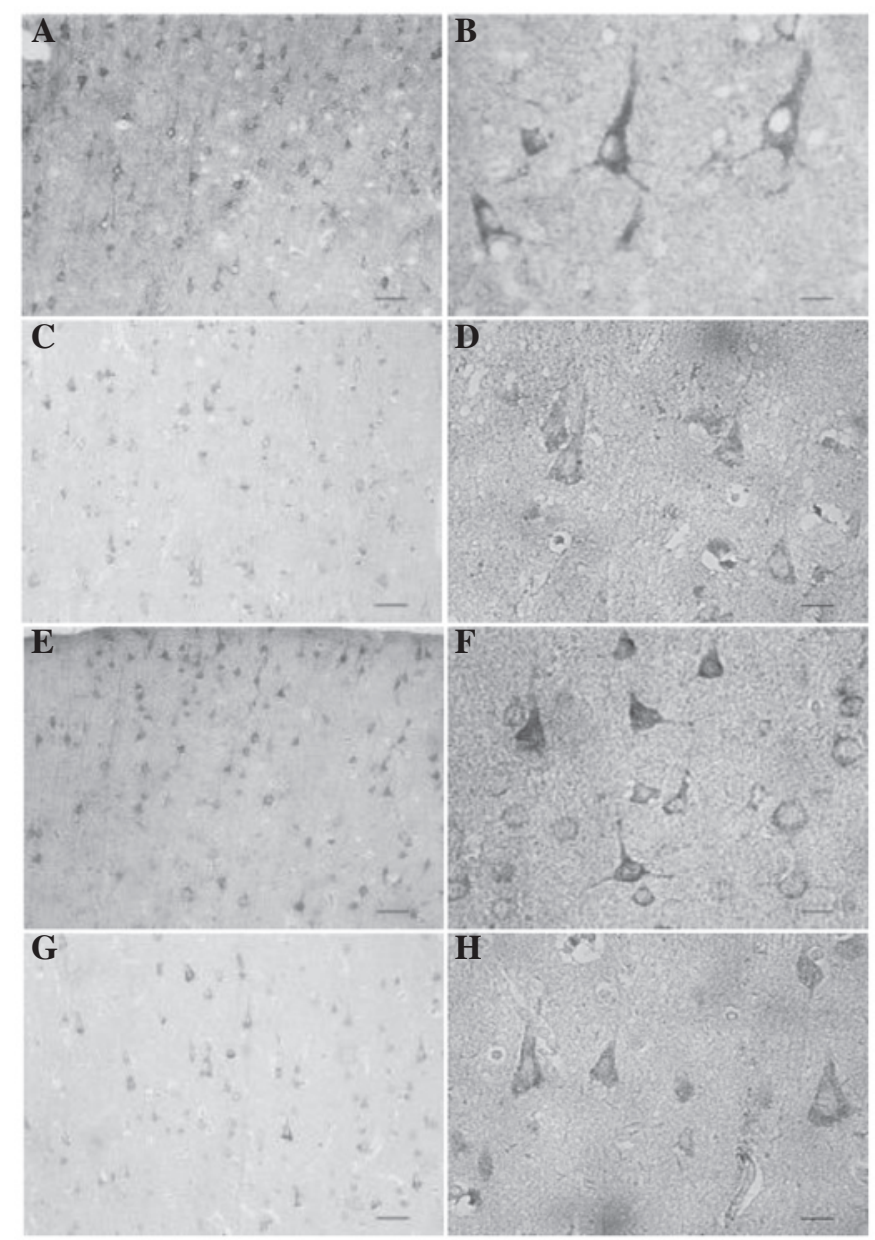

Figure 1. Microphotographs of immunohistochemistry. Distinct localization of Panx1 and Panx2 in layer III of control (A, B, E, F) and epileptic (C, D, G, $\mathrm{H})$ temporal lobe cortex. The pyramidal cell bodies highly expressed Panx1 and Panx2 (A-D) in layer III of the temporal lobe in both groups. Scale bar, (A, C, E and G) $50 \mu \mathrm{m}$; (B, D, F and H) $10 \mu \mathrm{m}$. Pannexin, Panx.

the two groups of patients who donated tissues for the study (Table III). The TLE group included 27 male and 10 female patients with a mean age of 26.3 years (range, 17 to 39 years). The duration of epilepsy (time since onset of symptoms) ranged from 2 to 37 years (mean, 12.6 years). In total, 26 cases were categorized as partial seizures, secondarily gener-

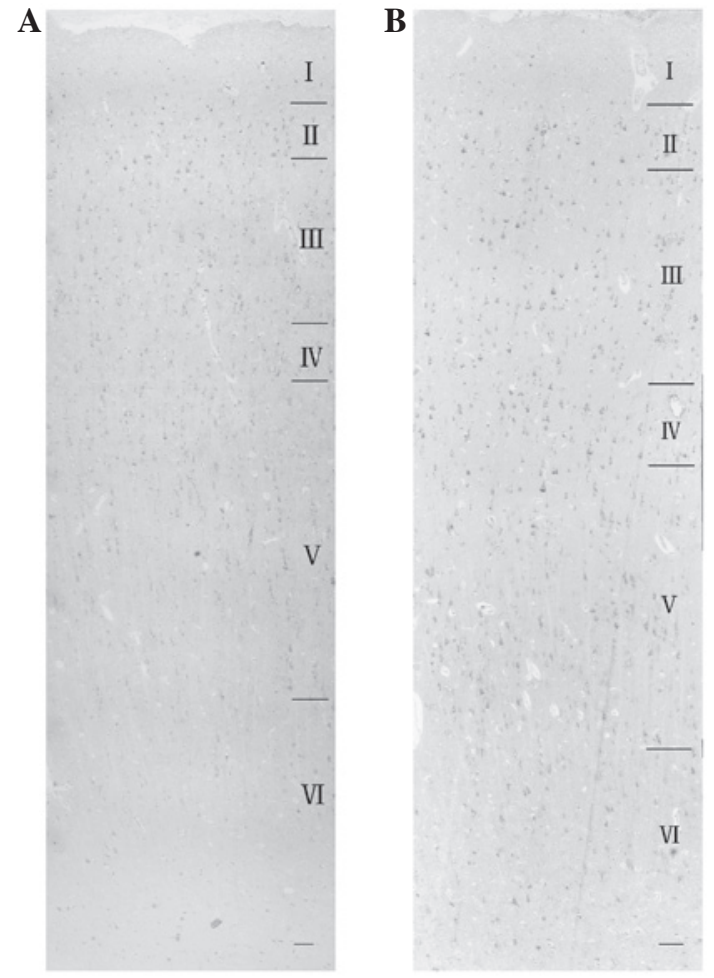

Figure 2. Distinct localization of (A) Panx1 and (B) Panx2 in the epileptic temporal lobe cortex. Panx 1 and Panx 2 proteins were expressed in all the six layers of the epileptic cortex in the temporal lobe formation. Expression was lowest in layer I. Scale bar, $100 \mu \mathrm{m}$ (A and B). Pannexin, Panx.

alized as tonic, clonic or tonic-clonic seizures, seven cases as complex partial seizures and four cases as other seizure types. The control group included six male and three female patients with brain trauma, between 10 and 40 years of age (mean age, 28.1 years).

Panx expression. Panx 1 and Panx 2 expression was detected in the temporal lobe cortex of patients with TLE and in the control tissues. In the control group, Panx1 and Panx 2 proteins were expressed predominantly in formation layers II and III of the cortex of the temporal lobe formation (Fig. 1). In the TLE group, Panx 1 and Panx 2 were expressed in all the six layers of the epileptic cortex, but less in layer I than in the other five layers (Fig. 2).

At a higher magnification, the cell bodies of pyramidal cells were shown to be immunoreactive for Panx1 (Fig. 1B and D) and Panx2 (Fig. 1F and H) in layer III. In the TLE group, the nuclei of several small-sized cells exhibited moderate immunoreactivity (Fig. 1D and F).

The expression level of Panx protein in the brain tissue of the TLE patients was subject to semi-quantitative analysis using western blotting (Fig. 3A). The variance between the two groups of patients was examined by calculating the ratio of the optical density of Panx to $\beta$-actin (Fig 3B and C). The Panx $1 / \beta$-actin ratio was $0.80 \pm 0.01$ for the TLE samples and $0.39 \pm 0.08$ for the control samples $(\mathrm{P}<0.05)$. Thus strong upregulation of Panx1 immunoreactivity was present in all samples from TLE patients compared with the control group.

The Panx $2 / \beta$-actin ratio of the TLE samples was $0.67 \pm 0.02$ and the corresponding ratio for the control samples was 
A

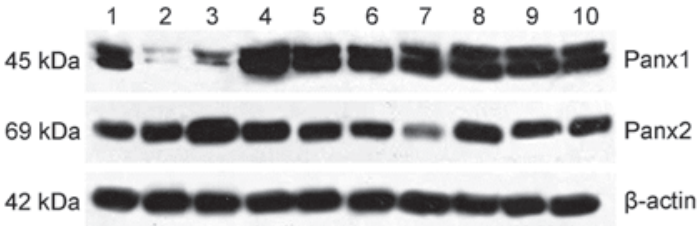

B

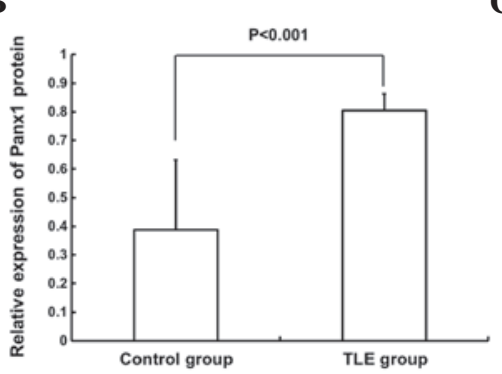

C

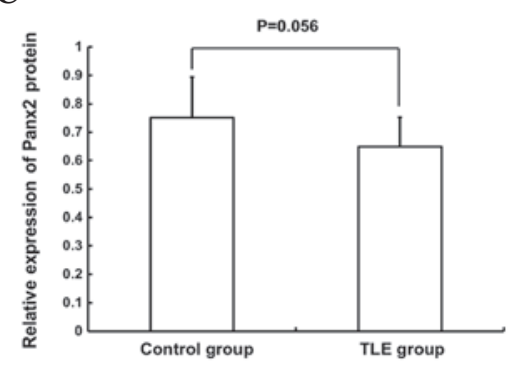

Figure 3. Semi-quantitative western blot analysis of Panx expression in the temporal lobe tissue of TLE patients. (A) Lanes 1-3 indicate protein levels in the control tissues, and lanes 4-10 indicate the protein levels of TLE patients. Immunoblot signals were developed by enhanced chemiluminescence. The two bands are likely to be the 43 and $47 \mathrm{kDa}$ isoforms of Panx1 present in mouse heart tissue lysate although this was not specifically investigated. (B and C) The average ratio of Panx to $\beta$-actin for each group of patients. Error bars are the SD. Pannexin, Panx.

$0.75 \pm 0.05$. This difference was not statistically significant $(\mathrm{P}>0.05)$.

\section{Discussion}

In the present study, the expression patterns of Panx 1 and Panx2 in the brain tissues of epileptic patients and controls were compared. To the best of our knowledge, this was the first time changes in Panx expression have been studied in human epileptic brains.

Panx1 and Panx2 are expressed in the central nervous system $(1,4,9,10)$. The expression of Panx1, but not of Panx2, in Xenopus oocytes forms functional hemichannels $(1,4)$. In addition, coinjection of these two Panx RNAs has been shown to result in hemichannels with functional properties that vary from those formed by Panx1 only. The functional characteristics of homomeric Panx1 versus heteromeric Panx1/Panx2 channels, and the varying expression patterns of Panx1 and Panx 2 in the brain, indicate that Panx form cell type-specific channels that have distinct properties and that may serve different functions.

In the present study, it was demonstrated that the Panx1 and Panx 2 proteins were expressed predominantly in layers II and III of the cortex in the control group. Previous studies have shown that Panx 1 and Panx $2 \mathrm{mRNAs}$ are present in all regions of the cerebral cortex of adult rats; however, the intensity of expression varies in the individual layers. For example, cells in layers II/III and V have been shown to exhibit a stronger signal for Panx mRNAs than those in layers I, IV and VI (4). This was not quite consistent with the results of the present study in the control groups. This apparent difference between the results was likely to be due to two reasons. The first was that the subjects studied varied; one subject was a rat while the other was human. The second reason may be due to different methods being adopted; the previous study conducted in situ hybridization in an animal, while the present study used immunohistochemistry and western blotting in patients.
Cortical neurons are important in epilepsy. Layers III, V and VI of the cortex are the layers in which pyramidal cells are predominantly distributed and from which association fibers arise, and layer IV is the main target of thalamocortical afferents from thalamic type $C$ neurons and from intra-hemispheric corticocortical afferents. Increased excitability and inward rectification in layer $\mathrm{V}$ cortical pyramidal neurons have been recorded in epileptic mutant mice (11). In chronically injured epileptogenic rats, excitatory synaptic connectivity is enhanced in layer $\mathrm{V}$ pyramidal neurons. This is accompanied by increased total axonal length and increased density of synaptic boutons (12). There is also evidence that epileptic discharges may be initiated from layer V and VI neurons (13). In the present study, Panx1 and Panx 2 were expressed in all six layers of the epileptic cortex, with the lowest expression observed in layer I. Ectopic expression of Panx in the epileptic cortex indicates that Panx proteins may be involved in the function of neurons in layers IV, V and VI of the epileptic cortex.

The study of Panx1, as shown by western blot analysis, confirmed previous results obtained with $\mathrm{Co}^{2+}$-treated brain slices (14). However the Panx 2 findings are at variance. With the $\mathrm{Co}^{2+}$-treated brain slices there was a 1.5 -fold increase in Panx1 and a 1.4-fold increase in Panx 2 mRNA; significant post-translational modifications of Panx1 protein were also observed after $\mathrm{Co}^{2+}$ treatment. While the present study identified significantly increased Panx1 levels in the TLE group, the change in Panx 2 protein levels was not significant. With the exception of the difference between the species of the subjects studied and the research methods, this inconsistency was most likely due to the choice of the brain slice model vs. TLE patients to detect changes in Panx expression, which possibly reflects differences in the functional mechanisms of Panx in TLE and in acute seizure activity induced by $\mathrm{Co}^{2+}$. Therefore, further studies are required to verify the most likely mechanism.

These experiments provide the first evidence for the expression of Panx protein in temporal lobe tissues of patients with 
TLE. The present study observed increased Panx1 expression and the ectopic expression of Panx1 and Panx2 in temporal lobe tissue in epilepsy. These results may be associated with the functional changes of cells in the epileptic cortex. The results support the conclusion that Panx channels are important in the formation of epilepsy.

\section{Acknowledgements}

This study was supported by grants from the National Natural Science Foundation of China (grant nos. 81071048, 81100967 and 81000553) and the Specialized Research Fund for the Doctoral Program of Higher Education (grant no. 20110162120002). The authors would like to thank the patients and their families for their participation in this study. The authors would also like to sincerely thank The Second Xiangya Hospital, The Second People's Hospital of Hunan Province for their assistance in the brain tissue procurement, and the local ethics committee for their support. Moreover, the authors are grateful to Jinghui Liang and Zhiguo $\mathrm{Wu}$ for their technical assistance.

\section{References}

1. Panchin Y, Kelmanson I, Matz M, Lukyanov K, Usman N and Lukyanov S: A ubiquitous family of putative gap junction molecules. Curr Biol 10: R473-474, 2000.

2. Bruzzone R, Hormuzdi SG, Barbe MT, Herb A and Monyer H: Pannexins, a family of gap junction proteins expressed in brain Proc Natl Acad Sci USA 100: 13644-13649, 2003.

3. Bruzzone R, White TW and Paul DL: Connections with connexins: the molecular basis of direct intercellular signaling. Eur J Biochem 238: 1-27, 1996.
4. White TW and Paul DL: Genetic diseases and gene knockouts reveal diverse connexin functions. Annu Rev Physiol 61: 283-310, 1999

5. Vogt A, Hormuzdi SG and Monyer H: Pannexin1 and Pannexin2 expression in the developing and mature rat brain. Brain Res Mol Brain Res 141: 113-120, 2005.

6. Thompson RJ, Jackson MF, Olah ME, et al: Activation of pannexin-1 hemichannels augments aberrant bursting in the hippocampus. Science 322: 1555-1559, 2008.

7. Kawamura M Jr., Ruskin DN and Masino SA: Metabolic autocrine regulation of neurons involves cooperation among pannexin hemichannels, adenosine receptors, and KATP channels. J Neurosci 30: 3886-3895, 2010.

8. No authors listed: Proposal for Revised Clinical and Electroencephalographic Classification of Epileptic Seizures. Epilepsia 22, 489-501, 1981.

9. Yuan J, Wang LY, Li JM, et al: Altered expression of the small guanosine triphosphatase RhoA in human temporal lobe epilepsy. J Mol Neurosci 42: 53-58, 2010.

10. Zappalà A, Cicero D, Serapide MF, et al: Expression of pannexin1 in the CNS of adult mouse: cellular localization and effect of 4-aminopyridine-induced seizures. Neuroscience 141: 167-178, 2006.

11. Zappalà A, Li Volti G, Serapide MF, et al: Expression of pannexin 2 protein in healthy and ischemized brain of adult rats. Neuroscience 148: 653-667, 2007.

12. Di Pasquale E, Keegan KD and Noebels JL: Increased excitability and inward rectification in layer $\mathrm{V}$ cortical pyramidal neurons in the epileptic mutant mouse Stargazer. J Neurophysiol 77: 621-631, 1997.

13. Jin X, Prince DA and Huguenard JR: Enhanced excitatory synaptic connectivity in layer $\mathrm{v}$ pyramidal neurons of chronically injured epileptogenic neocortex in rats. J Neurosci 26: 4891-4900, 2006.

14. Polack PO, Guillemain I, Hu E, Deransart C, Depaulis A and Charpier S: Deep layer somatosensory cortical neurons initiate spike-and-wave discharges in a genetic model of absence seizures. J Neurosci 27: 6590-6599, 2007.

15. Mylvaganam S, Zhang L, Wu C, et al: Hippocampal seizures alter the expression of the pannexin and connexin transcriptome. J Neurochem 112: 92-102, 2010. 